\title{
GROWTH RATES OF ALGEBRAS, III:
}

\author{
FINITE SOLVABLE ALGEBRAS
}

\author{
KEITH A. KEARNES, EMIL W. KISS, AND ÁGNES SZENDREI \\ Dedicated to the memory of Ervin Fried
}

\begin{abstract}
We investigate how the behavior of the function $d_{\mathbf{A}}(n)$, which gives the size of a least size generating set for $\mathbf{A}^{n}$, influences the structure of a finite solvable algebra $\mathbf{A}$.
\end{abstract}

\section{INTRODUCTION}

The growth rate (or the $d$-function) of a finite algebra $\mathbf{A}$ is $d_{\mathbf{A}}(n)=$ the least size of a generating set for $\mathbf{A}^{n}$. For a solvable group, this rate is always linear in $n$. On the other hand, unary algebras (which are also solvable) have exponential growth rate. In this paper we investigate the relationship between the growth rate of $\mathbf{A}$ and its structural properties in the case, when $\mathbf{A}$ is finite and solvable.

It turns out that stronger abelianness properties yield a closer relationship between various growth-restricting conditions. For example, if the variety generated by $\mathbf{A}$ is abelian, or if $\mathbf{A}$ is a subdirect product of simple abelian algebras, then the growth rate is non-exponential if and only if $\mathbf{A}$ has a Maltsev term, in which case the growth rate is linear. This result does not hold for nilpotent (hence for solvable) algebras.

To define the abelianness properties investigated in this paper the commutator from Chapter 3 of [2] is used. Some fluency in tame congruence theory is required to understand the proofs. The properties are the following:

(1) A is solvable,

(2) $\mathbf{A}$ is (left) nilpotent (see [3]),

(3) A is abelian,

(4) $\mathbf{A}$ is a subdirect product of simple abelian algebras, or

(5) A generates an abelian variety.

1991 Mathematics Subject Classification. 08A40 (08A55, 08B05).

Key words and phrases. Growth rate, solvable algebra, nilpotent algebra, abelian algebra, Maltsev term, pointed cube term, tame congruence theory.

This material is based upon work supported by the Hungarian National Foundation for Scientific Research (OTKA) grant no. K83219, and K104251. 
It is not hard to show from the definitions that these properties are related by the implications $(4) \Rightarrow(3) \Rightarrow(2) \Rightarrow(1)$ and $(5) \Rightarrow(3)$. It is also not too hard to show that no other implications hold except those that are formal consequences of these. (To verify this latter statement, one must find examples showing that $(1) \not \Rightarrow(2),(2) \not \Rightarrow(3)$, $(3) \nRightarrow(4),(3) \nRightarrow(5),(4) \nRightarrow(5)$, and $(5) \nRightarrow(4)$, and for many of these there exists an example that is a group. In the two situations where there is no group example, $(3) \nRightarrow(5)$ and $(4) \nRightarrow(5)$, the algebra from Example 8.7 of [5] shows that a simple abelian algebra can generate a nonabelian variety.)

Now consider the following six growth-restricting conditions:

(i) A has a Maltsev polynomial.

(ii) A has a pointed cube polynomial (see Definition 2.6).

(iii) $\mathbf{A}$ is a spread of its type $\mathbf{2}$ minimal sets (see Definition 5.11).

(iv) $d_{\mathbf{A}}(n) \in O(n)$.

(v) $d_{\mathbf{A}}(n) \notin 2^{\Omega(n)}$.

(vi) No finite power $\mathbf{A}^{n}$ has a nontrivial strongly abelian homomorphic image.

For arbitrary finite algebras, these conditions are related by the implications (i) $\Rightarrow$ (iv), (i) $\Rightarrow($ ii $) \Rightarrow(v)$, and (iii) $\Rightarrow($ iv $) \Rightarrow(v) \Rightarrow($ vi) and no implications hold that are not consequences of these, as we show in Theorem 2.12 .

In this paper we show that as we assume stronger and stronger hypotheses from the list (1)-(5), the conditions (i)-(vi) gradually become equivalent to one another. The following theorem summarizes our main results.

Theorem 1.1. Let $\mathbf{A}$ be a finite algebra.

(1) If $\mathbf{A}$ is solvable, then ( $\mathrm{i}) \Rightarrow$ (iii) by Corollary 5.6 and (ii) $\Rightarrow$ (iv) by Theorem 4.1 .

(2) If $\mathbf{A}$ is left nilpotent, then (ii) $\Rightarrow$ (i) by Theorem 6.1 and (vi) $\Rightarrow$ (iii) by Theorem 6.3. Therefore (i) and (ii) are equivalent, (iii), (iv), (v) and (vi) are equivalent, and the first group of equivalent conditions implies the second group. The same implications (and no others, cf. Example 8.3) hold under the stronger assumption that $\mathbf{A}$ is abelian.

(3) If $\mathbf{A}$ is a subdirect product of simple abelian algebras, then (iv) $\Rightarrow$ (i) by Theorem 8.1. Therefore all six conditions are equivalent for $\mathbf{A}$.

(4) If $\mathbf{A}$ generates an abelian variety, then (iii) $\Rightarrow$ (i) by Theorem 7.1. Therefore all six conditions are equivalent for $\mathbf{A}$.

These results are expressed diagrammatically in Section 9.

Our theorem completely determines the relationships between the growth-restricting conditions (i)-(vi) for nilpotent and abelian algebras, but some questions remain open for solvable algebras. We do know that (i) $\Rightarrow($ ii $) \Rightarrow($ iv $) \Rightarrow(v) \Rightarrow($ vi) and (i) $\Rightarrow$ (iii) $\Rightarrow$ (iv) for solvable algebras, and we also know that (iii) $\nRightarrow$ (ii) $\nRightarrow$ (i). We know nothing else about the implications between these properties for finite solvable algebras that are not nilpotent. 
The original purpose of our investigation was to show that the $d$-function of a finite solvable algebra grows at a linear or exponential rate. We were not able to prove or refute this statement. We considered the other conditions in order to better understand what might force the $d$-function of a solvable algebra into $O(n)$ or $2^{\Omega(n)}$. If, for example, one could now show that (vi) $\Rightarrow$ (iii) for finite solvable algebras, then one would have that the $d$-functions of such algebras grow linearly or exponentially.

\section{Preliminaries}

2.1. Notation. We use Big Oh notation. If $f$ and $g$ are real-valued functions defined on some subset of the real numbers, then $f \in O(g)$ means that there are positive constants $M$ and $N$ such that $|f(x)| \leq M|g(x)|$ for all $x>N$. We write $f \in \Omega(g)$ to mean that there are positive constants $M$ and $N$ such that $|f(x)| \geq M|g(x)|$ for all $x>N$. Finally, $f \in \Theta(g)$ means that both $f \in O(g)$ and $f \in \Omega(g)$ hold.

\subsection{Easy Estimates.}

Theorem 2.1 (Theorem 2.2.1 of [7]). Let $\mathbf{A}$ be an algebra.

(1) $d_{\mathbf{A}^{k}}(n)=d_{\mathbf{A}}(k n)$.

(2) If $\mathbf{B}$ is a homomorphic image of $\mathbf{A}$, then $d_{\mathbf{B}}(n) \leq d_{\mathbf{A}}(n)$.

(3) If $\mathbf{B}$ is an expansion of $\mathbf{A}$ (with operations; equivalently, if $\mathbf{A}$ is a reduct of $\mathbf{B})$, then $d_{\mathbf{B}}(n) \leq d_{\mathbf{A}}(n)$.

(4) (From [12]) If $\mathbf{B}$ is the expansion of $\mathbf{A}$ obtained by adjoining all constants, then

$$
d_{\mathbf{A}}(n)-d_{\mathbf{A}}(1) \leq d_{\mathbf{B}}(n) \leq d_{\mathbf{A}}(n)
$$

Theorem 2.2 (From Theorem 2.2.2 of [7]). If $\mathbf{A}$ is a finite algebra of more than one element and $n>0$, then

$$
\left\lceil\log _{|A|}(n)\right\rceil \leq d_{\mathbf{A}}(n) \leq|A|^{n}
$$

Recall that the free spectrum of a variety $\mathcal{V}$ is the function $f_{\mathcal{V}}(n):=\left|F_{\mathcal{V}}(n)\right|$ whose value at $n$ is the cardinality of the $n$-generated free algebra in $\mathcal{V}$.

Theorem 2.3 (From Theorem 2.2.4 of [7]). If $\mathbf{A}$ is a nontrivial finite algebra and $f_{\mathcal{V}}$ is the free spectrum of the variety $\mathcal{V}=\mathcal{V}(\mathbf{A})$, then

(1) if $f_{\mathcal{V}}(n) \in O\left(n^{k}\right)$ for some fixed $k \in \mathbb{Z}^{+}$, then $d_{\mathbf{A}}(n) \in 2^{\Theta(n)}$;

(2) if $f_{\mathcal{V}}(n) \in 2^{O(n)}$, then $d_{\mathbf{A}}(n) \in \Omega(n)$.

Corollary 2.4 (Corollary 2.2.5 of [7]). Let A be a nontrivial finite algebra and let $\mathbf{B}$ be a nontrivial homomorphic image of $\mathbf{A}^{k}$ for some $k$.

(1) If $\mathbf{B}$ is strongly abelian (or even just strongly rectangular), then $d_{\mathbf{A}}(n) \in 2^{\Theta(n)}$.

(2) If $\mathbf{B}$ is abelian, then $d_{\mathbf{A}}(n) \in \Omega(n)$. 
Theorem 2.5 (Theorem 2.2.6 of [7]). If $\mathbf{A}^{2}$ is a finitely generated affine algebra, then $d_{\mathbf{A}}(n) \in O(n)$. If, moreover, $\mathbf{A}$ is finite and has more than one element, then $d_{\mathbf{A}}(n) \in \Theta(n)$.

2.3. Basic relationships among conditions (i) $-(\mathbf{v i})$. Recall that a polynomial $F(x, y, z)$ of $\mathbf{A}$ is a Maltsev polynomial if $F(x, y, y) \approx x \approx F(y, y, x)$ holds in $\mathbf{A}$. In [7], this well known concept is generalized to the following:

Definition 2.6. A term $F\left(x_{1}, \ldots, x_{m}\right)$ is an $m$-ary, p-pointed, $k$-cube term for $\mathbf{A}$ if there is a $k \times m$ matrix $M$ consisting of variables and $p$ distinct constant symbols, with every column of $M$ containing a symbol different from $x$, such that $\mathbf{A}$ satisfies

$$
F(M) \approx\left(\begin{array}{c}
x \\
\vdots \\
x
\end{array}\right) .
$$

For example,

$$
F\left(\begin{array}{lll}
x & y & y \\
y & y & x
\end{array}\right) \approx\left(\begin{array}{l}
x \\
x
\end{array}\right)
$$

is a way to express that $F$ is a Maltsev term in the form (2.1) with $m=3, p=0$ and $k=2$.

The main results from [7] about the restriction on growth imposed by a pointed cube term now follow.

Theorem 2.7 (Theorem 5.2.1 of [7]). Let $\mathbf{A}$ be an algebra with an m-ary, p-pointed, $k$-cube term, with at least one constant symbol appearing in the cube identities (that is, $p \geq 1$ ). If $\mathbf{A}^{p+k-1}$ is finitely generated, then all finite powers of $\mathbf{A}$ are finitely generated and $d_{\mathbf{A}}(n)$ is bounded above by a polynomial of degree at most $\log _{w}(m)$, where $w=2 k /(2 k-1)$.

Corollary 2.8 (Corollary 5.2.4 of [7]). If $\mathbf{A}^{k}$ is a finitely generated algebra with a 0 -pointed or 1-pointed $k$-cube term, then $d_{\mathbf{A}}(n) \in O\left(n^{k-1}\right)$.

Definition 2.9. An $m$-ary, $p$-pointed, $k$-cube term for the constant expansion of $\mathbf{A}$ is called an $m$-ary, p-pointed, $k$-cube polynomial for $\mathbf{A}$.

Remark 2.10. An algebra has a Maltsev polynomial (or a pointed cube polynomial) if and only if its constant expansion has a Maltsev term (or pointed cube term). Passing to the constant expansion does not affect the growth rate significantly, as noted in Theorem 2.1 (4). Therefore, the last two results hold for polynomials as well as for terms. In fact, replacing "term" with "polynomial" in Theorem 2.7 and Corollary 2.8 yields correct statements.

In the literature, a 0-pointed cube term is called a cube-term. Note that a $p$-pointed cube term is not necessarily a cube-term even if all constants are unary terms. 
Theorem 2.11 (From Theorem 5.4.1 of [7]). Let $\mathbf{A}$ be an algebra with $|A|>1$ whose $d$-function assumes only finite values. There is an algebra $\mathbf{B}$ such that $d_{\mathbf{B}}(n)=d_{\mathbf{A}}(n)$ for all $n$, where $\mathbf{B}$ does not have a pointed cube polynomial.

For the other concepts appearing in the conditions (i)-(vi), we direct the reader to Definition 5.1 for "spread" and to [2] for "minimal set" and "strongly abelian".

We can now establish which implications between conditions (i)-(vi) from the Introduction hold for all finite algebras.

Theorem 2.12. For arbitrary finite algebras, the conditions

(i) A has a Maltsev polynomial.

(ii) A has a pointed cube polynomial.

(iii) A is a spread of its type $\mathbf{2}$ minimal sets.

(iv) $d_{\mathbf{A}}(n) \in O(n)$.

(v) $d_{\mathbf{A}}(n) \notin 2^{\Omega(n)}$.

(vi) No finite power $\mathbf{A}^{n}$ has a nontrivial strongly abelian homomorphic image.

are related by the implications

$$
(i) \Rightarrow(i v), \quad(i) \Rightarrow(i i) \Rightarrow(v), \quad \text { and } \quad(i i i) \Rightarrow(i v) \Rightarrow(v) \Rightarrow(v i) .
$$

The following non-implications can be established with finite counterexamples:

$$
(v i) \nRightarrow(v) \nRightarrow(i v) \nRightarrow(i i i) \nRightarrow(i i) \nRightarrow(i v) \nRightarrow(i i) \text { and }(i) \nRightarrow(\text { iii }) .
$$

These facts imply that the implications that hold are only those indicated in the following diagram and their consequences:

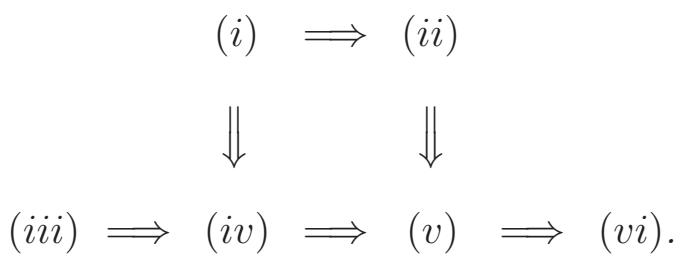

We shall prove this theorem in Section 9

\section{A NEW CHARACTERIZATION OF SOLVABILITY}

In this section we show how elementary translations coming from idempotent polynomials characterize solvability.

Definition 3.1. Let $\mathbf{A}$ be an algebra and $p$ an idempotent polynomial of $\mathbf{A}$ (that is, $p(x, x, \ldots, x)=x$ for every $x \in A)$. The translation-digraph $\operatorname{Tr}(p)$ of $p$ has vertex set $A$, and directed edges of the form $\left(c, c^{\prime}\right)=(p(c, c, \ldots, c), p(c, \ldots, c, d, c, \ldots, c))$, where $c, d \in A$. (The element $d$ occurs in exactly one of the arguments of $p$, but it can be any of the arguments.) 
Recall that a neighborhood $U$ of an algebra $\mathbf{A}$ is the range of an idempotent unary polynomial of $\mathbf{A}$, that is, $U=e(A)$ for a unary polynomial $e$ satisfying $e(e(x))=e(x)$ for every $x \in A$.

Theorem 3.2. Let $\mathbf{A}$ be a finite algebra. Then $\mathbf{A}$ is solvable if and only if for every neighborhood $U$ of $\mathbf{A}$, and every idempotent polynomial $p$ of the induced algebra $\left.\mathbf{A}\right|_{U}$, the directed graph $\operatorname{Tr}(p)$ is strongly connected.

The proof of Theorem 3.2 is included at the end of this section. The essential part of the proof is to establish the theorem for classes of solvable minimal congruences.

Lemma 3.3. Let $\mathbf{S}$ be a finite, simple, abelian algebra and $p$ an idempotent polynomial of $\mathbf{S}$. Then $\operatorname{Tr}(p)$ is strongly connected.

Proof. Since $S$ is connected by traces, it is sufficient to prove that for every trace $N$ and every $a, b \in N$ there is a directed path in $\operatorname{Tr}(p)$ connecting $a$ to $b$. Consider the multitraces with respect to $N$, that is, all sets of the form $q(N, N, \ldots, N)$, where $q$ is any polynomial of $\mathbf{S}$. Choose a multitrace $M$ that contains $N$ and is maximal under inclusion. Then $p(M, \ldots, M)$ contains $M$ (and therefore $N$ ), since $p$ is idempotent, and is a multitrace, so $p(M, \ldots, M)=M$. Theorem 3.10 of [9] shows that the induced algebra $\left.\mathbf{S}\right|_{M}$ is polynomially equivalent to the full matrix power $\left(\left.\mathbf{S}\right|_{N}\right)^{[k]}$ for some $k$ (more precisely, $\left.\mathbf{S}\right|_{M}$ is isomorphic to an algebra on $N^{k}$ that is polynomially equivalent to $\left.\left(\left.\mathbf{S}\right|_{N}\right)^{[k]}\right)$.

Consider first the case, when the type of $\mathbf{S}$ is $\mathbf{1}$. Let $a$ correspond to the vector $\left(a_{1}, \ldots, a_{k}\right)$ and $b$ correspond to $\left(b_{1}, \ldots, b_{k}\right)$. We may assume that $a$ and $b$ differ only in one coordinate, and, to simplify the notation, that this is the first coordinate. That is, $a_{i}=b_{i}$ for $i \geq 2$. Indeed, if such pairs can be connected by a directed path, then by changing only one coordinate at a time we can connect $a$ to $b$.

By the definition of a matrix power, the operation of $p$ induced on $M$ can be represented as follows:

$$
p\left(\mathbf{x}^{1}, \ldots, \mathbf{x}^{\ell}\right)=\left(\begin{array}{c}
p_{1}\left(x_{1}^{1}, x_{1}^{2}, \ldots, x_{i}^{j}, \ldots, x_{k}^{\ell}\right) \\
\vdots \\
p_{k}\left(x_{1}^{1}, x_{1}^{2}, \ldots, x_{i}^{j}, \ldots, x_{k}^{\ell}\right)
\end{array}\right),
$$

where $\mathbf{x}^{j}$ is (the transpose of) $\left(x_{1}^{j}, \ldots, x_{k}^{j}\right)$ and each $p_{i}$ is a $k \ell$-ary operation of $\left.\mathbf{S}\right|_{N}$. Hence each $p_{i}$ is essentially unary.

We use that $p$ is idempotent. Consider $p(\mathbf{x}, \ldots, \mathbf{x})$ and move the first coordinate $x_{1}$ of $\mathbf{x}$ in $N$ while keeping all other coordinates fixed. Then the value of $p_{1}$ must change, so $p_{1}$ must depend on one of the variables $x_{1}^{1}, x_{1}^{2}, \ldots, x_{1}^{\ell}$, and therefore on no other variable. Let this variable be $x_{1}^{j}$. The fact that $p$ is idempotent shows that $p_{1}\left(x_{1}^{1}, x_{1}^{2}, \ldots, x_{k}^{\ell}\right)=x_{1}^{j}$. An analogous statement holds for all other rows, in particular, each $p_{i}$ is a projection, hence it is idempotent. Substitute $b=\left(b_{1}, \ldots, b_{k}\right)$ 
to the $j$-th variable of $p$ and $a=\left(a_{1}, \ldots, a_{k}\right)$ to all other variables. Then in the first row we get $b_{1}$, and in the $i$-th row we get $a_{i}=b_{i}$ (if $i \geq 2$ ). Therefore the result of the operation is $b$, and so there is an edge $(a, b)$ in $\operatorname{Tr}(p)$.

If the type of $\mathbf{S}$ is $\mathbf{2}$, then $\left.\mathbf{S}\right|_{N}$ is polynomially equivalent to a 1-dimensional vector space over a field $\mathbf{F}$, and $\left(\left.\mathbf{S}\right|_{N}\right)^{[k]}$ is polynomially equivalent to the module $N^{k}$ over $\mathbf{F}^{k \times k}$, where the matrices act on $N^{k}$ by multiplication. Since $p$ is idempotent,

$$
p\left(\mathbf{x}^{1}, \ldots, \mathbf{x}^{\ell}\right)=M_{1} \mathbf{x}^{1}+\ldots+M_{\ell} \mathbf{x}^{\ell}
$$

holds for some matrices $M_{i} \in \mathbf{F}^{k \times k}$ satisfying that their sum is the identity matrix.

Notice that for any $\mathbf{x}, \mathbf{y} \in N^{k}$ we have

$$
M_{1}(\mathbf{x}+\mathbf{y})+M_{2} \mathbf{x}+\ldots+M_{\ell} \mathbf{x}=\mathbf{x}+M_{1} \mathbf{y},
$$

so $\left(\mathbf{x}, \mathbf{x}+M_{1} \mathbf{y}\right)$ is an edge of $\operatorname{Tr}(p)$, and similarly, $\left(\mathbf{x}, \mathbf{x}+M_{i} \mathbf{y}\right)$ is an edge, too, for every $1 \leq i \leq \ell$. Now let $\mathbf{a}, \mathbf{b} \in N^{k}$. Since

$$
M_{1}(\mathbf{b}-\mathbf{a})+\ldots+M_{\ell}(\mathbf{b}-\mathbf{a})=\mathbf{b}-\mathbf{a},
$$

we have

$$
\mathbf{a}+M_{1}(\mathbf{b}-\mathbf{a})+\ldots+M_{\ell}(\mathbf{b}-\mathbf{a})=\mathbf{b},
$$

and by our remark, this yields a path of $\ell$ edges in $\operatorname{Tr}(p)$ connecting $\mathbf{a}$ to $\mathbf{b}$.

Proof of Theorem 3.2. Let $A$ be a finite solvable algebra. We argue by induction on $|A|$. Let $U$ be a neighborhood of $\mathbf{A}$. Then the induced algebra $\left.\mathbf{A}\right|_{U}$ is solvable, too, so we can assume that $U=A$. Choose and fix an idempotent polynomial $p$ of $\mathbf{A}$. Let $\alpha$ be a minimal congruence of $\mathbf{A}$. By the induction assumption, $\mathbf{A} / \alpha$ is strongly connected with respect to $p / \alpha$. Thus if $a, b \in A$ are given, then there exist edges $\left(u_{1}, v_{1}\right), \ldots,\left(u_{k}, v_{k}\right)$ of $\operatorname{Tr}(p)$ such that $a \equiv_{\alpha} u_{1}, v_{k} \equiv_{\alpha} b$, and each $v_{i} \equiv_{\alpha} u_{i+1}$ for $1 \leq i \leq k-1$. Therefore it is sufficient to prove that each $\alpha$-class is strongly connected. Let $S$ be such a class. Then the induced algebra $\left.\mathbf{A}\right|_{S}$ is simple, abelian, and $\left.p\right|_{S}$ is a polynomial of this induced algebra, since $p$ is idempotent. Therefore Lemma 3.3 shows that $S$ is strongly connected indeed.

For the converse, suppose that $A$ is not solvable. Then there is a prime quotient $\langle\delta, \theta\rangle$ in $\operatorname{Con}(\mathbf{A})$ of nonabelian type. Let $U$ be a $\langle\delta, \theta\rangle$-minimal set. By [2], Lemma 2.10 and Theorems 4.15, 4.17, and 4.23, $U$ is a neighborhood, and there is a pseudo-meet operation $p$ on $\left.\mathbf{A}\right|_{U}$. This operation is idempotent, satisfies the identity $p(x, y)=p(x, p(x, y))$, and there is an element $1 \in U$ such that $p(1, x)=p(x, 1)=x$ for every $x \in U$. These properties imply that there is no nontrivial edge in $\operatorname{Tr}(p)$ whose endpoint is 1 , hence this graph is not strongly connected. Indeed, if $p(c, d)=1$, then

$$
1=p(c, d)=p(c, p(c, d))=p(c, 1)=c,
$$


and then $1=p(c, d)=p(1, d)=d$, so $c=d=1$. This calculation shows that any edge terminating at 1 must originate from 1 . Thus the proof of Theorem 3.2 is complete.

\section{Solvable algebras With a pointed Cube term}

Theorem 4.1. Let $\mathbf{A}$ be a finite, nontrivial solvable algebra that has a pointed cube term. Then $d_{\mathbf{A}}(n) \in \Theta(n)$. In fact, the algebra $\mathbf{A}^{n}$ is generated by those elements of $A^{n}$ that are constant with the possible exception of one component.

(This establishes for solvable algebras the extra implication (ii) $\Rightarrow$ (iv) among the conditions of Theorem 2.12, )

Proof. The quotient modulo any maximal congruence of $\mathbf{A}$ is abelian, so the combination of Corollary 2.4 (2) and Theorem 2.1 (2) shows that $d_{\mathbf{A}}(n) \in \Omega(n)$. Therefore it is sufficient to prove that $d_{\mathbf{A}}(n) \in O(n)$.

For a given $n$, let $G$ consist of those elements of $A^{n}$ that are constant with the possible exception of one component. The size of $G$ is $|A|+n|A|(|A|-1)$ for $n \geq 3$, which is linear in $n$. Thus it is enough to prove that $G$ is a generating set of $\mathbf{A}^{n}$. Let $\mathbf{B}$ be the subalgebra generated by $G$. Note that $B$ is symmetric under any permutation of coordinates. This will allow us to simplify notation in the proof below by rearranging coordinates at certain points.

We may (and shall) assume that our cube identities involve only the variable $x$, since all other variables can be replaced by a fixed constant from $A$.

We induct on $|A|$. Let $\alpha$ be a minimal congruence of $\mathbf{A}$. By our induction hypothesis, $(A / \alpha)^{n}$ is generated by $G /\left(\alpha^{n}\right)$, since this is the set of all sequences in $(A / \alpha)^{n}$ that are constant with the possible exception of one component. Therefore $B$ intersects each $\alpha^{n}$-class. Our proof shall proceed by "repairing" these representatives component by component.

For a given $1 \leq m \leq n$ call a sequence $\left(b_{1}, \ldots, b_{m}\right)$ of elements of $A$ complete, if for every $a_{m+1}, \ldots, a_{n}$ there exist elements $b_{m+1}, \ldots, b_{n}$ such that $\left(b_{1}, \ldots, b_{n}\right) \in B$ and $b_{i} \equiv_{\alpha} a_{i}$ for all $i>m$. We shall say informally that $\left(b_{1}, \ldots, b_{m}\right)$ can be extended into $B$ along $a_{m+1}, \ldots, a_{n}$. The argument in the previous paragraph shows that the empty sequence is complete (when $m=0$ ), and we endeavor to show that every element of $A^{n}$ is complete, which means that $B=A^{n}$. Therefore it is sufficient to prove the following.

Claim 4.2. Suppose that each element of $A^{m-1}$ is complete. Then each element of $A^{m}$ is complete.

Proof of claim. To set up notation, we permute the coordinates in the following way. We assume that $\left(b_{2}, \ldots, b_{m}\right)$ is complete and intend to show that $\left(c, b_{2}, \ldots, b_{m}\right)$ is complete for every $c$. 
Fix $a_{m+1}, \ldots, a_{n} \in A$. We have to extend $\left(c, b_{2}, \ldots, b_{m}\right)$ into $B$ along $a_{m+1}, \ldots, a_{n}$. The completeness of $\left(b_{2}, \ldots, b_{m}\right)$ allows us to find elements $b, b_{m+1}, \ldots, b_{n}$ such that $b \equiv_{\alpha} c, b_{j} \equiv_{\alpha} a_{j}$ for all $j>m$ and $\mathbf{b}=\left(b_{1}, \ldots, b_{n}\right) \in B$. In other words, $\left(b, b_{2}, \ldots, b_{m}\right)$ can be extended into $B$ along $a_{m+1}, \ldots, a_{n}$.

Let $S=b / \alpha=c / \alpha$. The induced algebra $\mathbf{S}=\left.\mathbf{C}\right|_{S}$ is simple and abelian. Consider a cube identity $F\left(x, \ldots, x, u_{1}, \ldots, u_{p}\right) \approx x$ (here we rearranged the variables appropriately and $u_{1}, \ldots, u_{p}$ are constants). For each constant $u_{j}$ extend $\left(b_{2}, \ldots, b_{m}\right)$ into $B$ along $u_{j}$ and $a_{m+1}, \ldots, a_{n}$. We get a vector $\mathbf{u}^{j} \in B$ whose first coordinate is denoted by $u_{j}^{\prime}$.

Consider the polynomials

$$
\begin{aligned}
& p\left(x_{1}, \ldots, x_{\ell}\right)=F^{\mathbf{A}}\left(x_{1}, \ldots, x_{\ell}, u_{1}, \ldots, u_{p}\right) \\
& q\left(x_{1}, \ldots, x_{\ell}\right)=F^{\mathbf{A}}\left(x_{1}, \ldots, x_{\ell}, u_{1}^{\prime}, \ldots, u_{p}^{\prime}\right) .
\end{aligned}
$$

The first polynomial is idempotent because of the cube identity above. Therefore $p(S, \ldots, S) \subseteq S$, and $p$ restricted to $S$ is a polynomial of $\mathbf{S}$. Since $u_{j} \equiv_{\alpha} u_{j}^{\prime}$, the polynomial $q$ can also be restricted to $S$.

The congruence $\alpha$ is abelian. This implies that $\pi(x)=q(x, x, \ldots, x)$ is a permutation of $S$. Indeed, if $q(s, \ldots s)=q(t, \ldots, t)$ for some $s, t \in S$, then using the term condition for $F$ (which can be applied, since $s \equiv_{\alpha} t$ and $u_{j} \equiv_{\alpha} u_{j}^{\prime}$ ) we get that $p(s, \ldots, s)=p(t, \ldots, t)$. Since $p$ is idempotent, we see that $s=t$. Thus $\pi$ is indeed a permutation. Let $o$ be the order of $\pi$ in the symmetric group on $S$ and $r\left(x_{1}, \ldots, x_{\ell}\right)=\pi^{o-1}\left(q\left(x_{1}, \ldots, x_{\ell}\right)\right)$. This is an idempotent polynomial of $\mathbf{S}$.

Lemma 3.3 can be applied to the polynomial $r$ to connect $b$ to $c$ by edges in $\operatorname{Tr}(r)$. We plan to go along this path and extend into $B$ step by step, so it is enough to show how to do a single edge. For the simplicity of notation we may assume that $(b, c)$ itself is an edge. That is, also by appropriately rearranging the arguments of $F$, that $r(d, b, \ldots, b)=c$ for some element $d \in S$. This means that $q(d, b, \ldots, b)=\pi(c)$.

Now choose a second cube identity, where the first variable is not $x$. We shall apply the term $F$ to a matrix $M$ whose elements we now describe. The number of rows is $n$ and the number of columns is the arity of $F$. We shall distinguish five types of columns of $M$, that is, arguments of $F$. The definitions of these types and the way we fill the entries of the matrix $M$ are shown on Figure 4.1 (page 10).

Applying $F$ to this matrix the first row yields $q(d, b, \ldots, b)=\pi(c)$. All other rows can be evaluated using the second cube identity. The result is $b_{j}$ for $2 \leq j \leq m$ and is $\alpha$-related to $a_{j}$ for $j>m$, since $b_{j}, b_{j}^{\prime} \equiv_{\alpha} a_{j}$.

This is almost the vector we are looking for, we only have to replace $\pi(c)$ by $c$ in the first component. In other words, it is sufficient to prove the following.

Subclaim 4.3. Suppose that $\left(\pi\left(c_{1}\right), c_{2}, \ldots, c_{n}\right) \in B$ holds for some vector where $c_{1} \in S$. Then $\left(c_{1}, c_{2}, \ldots, c_{n}\right) \in B$. 
The types of columns of $M$ :

(I) The first column/argument is the only one to have type I. There is $x$ in the first cube identity and a constant named $s$ in the second cube identity.

(II) Type II column/argument: both cube identities have $x$ in this argument.

(III) Type III: there is $x$ in the first cube identity, and a constant in the second. We exclude the first argument (which also has this property).

(IV) Type IV: there is a constant in the first cube identity, and $x$ in the second.

(V) Type V: there are constants in both cube identities.

The way to fill the columns of $M$ :

(V) In a type $\mathrm{V}$ argument, let $u_{k}$ be the constant in the first cube identity, and $w$ the constant in the second cube identity. Then put $u_{k}^{\prime}$ (defined above) into the first coordinate and $w$ to all other coordinates. (Of course for different columns these constants may differ.) This column is in $G$.

(IV) Into a type IV column, put the appropriate vector $\mathbf{u}^{j}$, also defined above. Recall that this vector extends $\left(b_{2}, \ldots, b_{m}\right)$ along $u_{j}, a_{m+1}, \ldots, a_{n}$ into $B$, and its first entry is $u_{j}^{\prime}$.

(III) For a type III argument, let $t$ be the constant appearing in the second cube identity. Put $b$ to the first coordinate and $t$ everywhere else. This column is in $G$.

(II) Put the vector $\mathbf{b} \in B$ defined above to all type II columns.

(I) To the first column (the only column of type $I$ ), put the element $d$ to the first coordinate, and $s$ (the constant in the first variable of the second cube identity) everywhere else. This is also in $G$.

$\begin{array}{ccccc}\text { I } & \text { II } & \text { III } & \text { IV } & \text { V } \\ d & b & b & u_{j}^{\prime} & u_{k}^{\prime} \\ s & b_{2} & t & b_{2} & w \\ s & b_{3} & t & b_{3} & w \\ \vdots & \vdots & \vdots & \vdots & \vdots \\ s & b_{m} & t & b_{m} & w \\ s & b_{m+1} & t & b_{m+1}^{\prime} & w \\ \vdots & \vdots & \vdots & \vdots & \vdots \\ s & b_{n} & t & b_{n}^{\prime} & w\end{array}$

Figure 4.1. The elements substituted into the five types of columns

Proof of subclaim. The equations in display (4.1) and $\pi(x)=q(x, x, \ldots, x)$ show that we have $\pi^{o-1}(x)=g\left(x, u_{1}^{\prime}, \ldots, u_{p}^{\prime}\right)$ for a term $g$ obtained from $F$ by composition, and if we replace each $u_{i}^{\prime}$ by $u_{i}$, then we get that $g\left(x, u_{1}, \ldots, u_{p}\right)=x$ (the $(o-1)$ st power 
of the identity map). The columns of the matrix

$$
\left(\begin{array}{cccc}
\pi\left(c_{1}\right) & u_{1}^{\prime} & \ldots & u_{p}^{\prime} \\
c_{2} & u_{1} & \ldots & u_{p} \\
\vdots & \vdots & \vdots & \vdots \\
c_{n} & u_{1} & \ldots & u_{p}
\end{array}\right)
$$

are in $B$ (for the first column we assumed this, and the others are in $G$ ). Applying the term $g$ to the rows we get that $\left(c_{1}, c_{2}, \ldots, c_{n}\right) \in B$, proving the subclaim and Claim 4.2. This completes the proof of Theorem 4.1, too.

\section{Spreads AND GROWTH RATE}

Definition 5.1. Let $\mathbf{A}$ be an algebra and $\mathcal{U}$ a collection of subsets of $A$. We say that a subset $S \subseteq A$ is a spread of $\mathcal{U}$ if there exists a polynomial $p$ of $\mathbf{A}$ and (not necessarily distinct) elements $U_{1}, \ldots, U_{k} \in \mathcal{U}$ such that $p\left(U_{1}, \ldots, U_{k}\right)=S$.

Lemma 5.2. Suppose that $\mathbf{A}$ is a finite algebra such that all constants are term operations, $p$ is a polynomial of $\mathbf{A}$ and $A=p\left(U_{1}, \ldots, U_{k}\right)$ holds for some subsets $U_{i} \subseteq A$. Then

$$
d_{\mathbf{A}}(n) \leq d_{\mathbf{U}_{1}}(n)+\ldots+d_{\mathbf{U}_{k}}(n),
$$

where $\mathbf{U}_{i}=\left.\mathbf{A}\right|_{U_{i}}$ are the algebras $\mathbf{A}$ induces on these sets.

Proof. For a given $n$ let $G_{i}$ be a smallest size generating set of the algebra $\mathbf{U}_{i}^{n}$. The set $G:=\bigcup_{i=1}^{k} G_{i}$ has size at most $d_{\mathbf{U}_{1}}(n)+\ldots+d_{\mathbf{U}_{k}}(n)$, and generates a subalgebra of $\mathbf{A}^{n}$ containing all sets of the form $U_{i}^{n}$. Since $p$ is a term operation of $\mathbf{A}$, and

$$
p^{\mathbf{A}^{n}}\left(U_{1}^{n}, \ldots, U_{k}^{n}\right)=\left(p^{\mathbf{A}}\left(U_{1}, \ldots, U_{n}\right)\right)^{n}=A^{n}
$$

we get that $G$ generates $\mathbf{A}^{n}$.

Corollary 5.3. If a finite algebra $\mathbf{A}$ is a spread of a family of subsets on which the induced algebras have Maltsev polynomials, then the growth rate of $\mathbf{A}$ is at most linear.

Proof. Use Corollary 2.8 and Lemma 5.2.

Several years ago, K. A. Kearnes, E. W. Kiss and M. A. Valeriote proved but did not publish the fact that any finite algebra with a Maltsev polynomial is covered by its $\langle\alpha, \beta\rangle$-minimal sets. This statement is a bit stronger than the statement that any finite algebra with a Maltsev polynomial is a spread of its minimal sets, so it is relevant here.

To introduce the terminology, if $\mathcal{U}$ and $\mathcal{V}$ are sets of neighborhoods of $\mathbf{A}$, then $\mathcal{U}$ covers $\mathcal{V}$ if for every $V \in \mathcal{V}$ there are neighborhoods $U_{1}, \ldots, U_{k} \in \mathcal{U}$, idempotent 
unary polynomials $e_{1}, \ldots, e_{k}, f$, and other polynomials $\lambda, \rho_{i}$ such that $f(A)=V$, $e_{i}(A)=U_{i}$ and

$$
\lambda\left(e_{1} \rho_{1}(x), \ldots, e_{k} \rho_{k}(x)\right)=f(x)
$$

for all $x \in A$. (Here the $e_{i}$ 's need not be distinct.) Equation (5.1) expresses $V$ as a retract of the product of the $U_{i}$ 's via polynomial maps $\lambda\left(x_{1}, \ldots, x_{k}\right)$ and $\left(e_{1} \rho_{1}(x), \ldots, e_{k} \rho_{k}(x)\right)$. It is not hard to see that if $\mathcal{U}$ covers $\mathcal{V}$ and $\mathcal{V}$ covers $\mathcal{W}$, then $\mathcal{U}$ covers $\mathcal{W}$. We say that $\mathcal{U}$ covers the algebra $\mathbf{A}$ if $\mathcal{U}$ covers the set $\{A\}$.

Lemma 2.10 of [2] guarantees that the minimal sets of a finite algebra are neighborhoods. If some set $\mathcal{U}$ of minimal sets covers $\mathbf{A}$, and this is witnessed as in (5.1) by polynomials $e_{i}, \lambda, \rho_{i}$ and $f=\mathrm{id}$, then equation (5.1) yields $\lambda\left(U_{1}, \ldots, U_{k}\right)=A$, so $A$ is a spread of the minimal sets in $\mathcal{U}$. Hence if $\mathbf{A}$ is covered by its minimal sets, then it is a spread of its minimal sets.

Theorem 5.4. If $\mathbf{A}$ is a finite algebra with a Maltsev polynomial, then $\mathbf{A}$ is covered by its $\langle\alpha, \beta\rangle$-minimal sets, where $\langle\alpha, \beta\rangle$ runs over all prime quotients of $\mathbf{C o n}(\mathbf{A})$.

Proof. Let $N \subseteq A$ be a neighborhood of $\mathbf{A}$ that is maximal for the property that $\left.\mathbf{A}\right|_{N}$ is covered by its minimal sets. If $N=A$, then we are done, so we assume otherwise and argue to a contradiction.

By Theorem 4.31 of [2], type 2 minimal sets in an algebra with a Maltsev polynomial are E-minimal. Hence a minimal set of a finite algebra with a Maltsev polynomial is nothing other than a set that is minimal under inclusion among nontrivial neighborhoods. Therefore the minimal sets of $\left.\mathbf{A}\right|_{N}$ are exactly the minimal sets of $\mathbf{A}$ that lie in $N$.

It is a basic fact of tame congruence theory (a consequence of Lemma 2.17 of [2]) that every finite algebra is the connected union of the traces of its minimal sets for congruences, hence if $N \neq A$ then there must exist $\alpha \prec \beta$ in $\operatorname{Con}(\mathbf{A})$ and an $\langle\alpha, \beta\rangle$-minimal set $U$ such that $U$ has a trace $T \subseteq U$ that properly overlaps $N$. (I.e., $T \cap N \neq \emptyset$, but $T \nsubseteq N$.) Choose $0 \in T \cap N$. Thus $U$ properly overlaps $N$ and $U \cap N$ contains an element 0 from (the body of) $U$. Let $m(x, y, z)$ denote a Maltsev polynomial of $\mathbf{A}$.

Claim 5.5. A has idempotent unary polynomials $e$ and $f$ such that

(1) $e(A)=U$ and $e(N)=\{0\}$, and

(2) $f(A)=N$ and $f(U)=\{0\}$.

Since $U$ and $N$ are neighborhoods there exist idempotent unary polynomials $e_{0}$ and $f_{0}$ such that $e_{0}(A)=U$ and $f_{0}(A)=N$. We show that these can be modified to have the extra properties in the claim.

Our first step is to construct $e$. As a first case, assume that $\left.e_{0} f_{0} \in \operatorname{Pol}_{1}(\mathbf{A})\right|_{U}$ is not a permutation of $U$. The polynomial $g(x)=e_{0} m\left(0, e_{0} f_{0}(x), e_{0}(x)\right)$ has the 
property that $g(A) \subseteq U$ and $g(N)=\{0\}$. Take $u \in T$ such that $(u, 0) \in \beta-\alpha$. Then $\left(e_{0} f_{0}(u), 0\right) \in \alpha$, and we get $(g(u), u) \in \alpha$. Since $(0, u) \in \beta-\alpha, g(0)=0$, and $g(u) \equiv u(\bmod \alpha)$ it follows that $g(\beta) \nsubseteq \propto \alpha$ and so $g$ is not collapsing on $U$. Therefore an appropriate power $e=g^{k}$ is an idempotent unary polynomial with range $U$ that collapses $N$ to $\{0\}$.

Now assume that $e_{0} f_{0}$ is a permutation of $U$. Then $U \simeq f_{0}(U)$, so $V:=f_{0}(U) \subseteq N$ is an $\langle\alpha, \beta\rangle$-minimal set contained in $N$ and containing 0 . Let $S=f_{0}(T)$ be the $\langle\alpha, \beta\rangle$ trace of $V$ containing 0 . Corollary 4.8 of [10] guarantees that there is an idempotent unary polynomial $e_{1}$ of $\mathbf{A}$ such that in the quotient $\mathbf{A} / \alpha$ we have $\bar{e}_{1}(A / \alpha)=U / \alpha$ and $\bar{e}_{1}(S / \alpha)=\{0 / \alpha\}$. Replacing $e_{1}$ by $e_{0} e_{1}$ if necessary we may assume that $e_{0} e_{1}=e_{1}$, so $e_{1}(A) \subseteq U$. Since $e_{1}$ maps $A$ into $U$ and it is the identity modulo $\alpha$ on $U$, it follows from the $\langle\alpha, \beta\rangle$-minimality of $U$ that $e_{1}(A)=U$. Now $e_{1} f_{0}$ is collapsing on $T$, so $e_{1} f_{0}$ is not a permutation of $U$. Thus we can repeat the argument of the first case using $e_{1}$ in place of $e_{0}$ to construct an idempotent unary polynomial $e$ such that $e(A)=U$ and $e(N)=\{0\}$.

Now, given $e$ and $f_{0}$ as above let $f(x)=f_{0} m\left(f_{0}(x), m\left(e(x), x, f_{0}(x)\right), 0\right)$. One calculates that $f(A) \subseteq N, f$ is the identity on $N$ (so $f$ is idempotent with range $N$ ), and $f(U)=\{0\}$. This completes the proof of the claim.

Now we finish the proof of the theorem. The polynomial $h(x)=m(e(x), 0, f(x))$ is the identity on $N \cup U$, so some iterate $h^{\ell}(x)$ is idempotent with range $h^{\ell}(A)=M \supsetneq N$. The equation

$$
h^{\ell} m\left(e\left(h^{\ell-1}(x)\right), 0, f\left(h^{\ell-1}(x)\right)\right)=h^{2 \ell}(x)=h^{\ell}(x)
$$

is an equation of type (5.1) for $\left.\mathbf{A}\right|_{M}$, showing that $\left.\mathbf{A}\right|_{M}$ is covered by its subneighborhoods $N$ and $U$. Because the covering relation is transitive, $\left.\mathbf{A}\right|_{M}$ is covered by $U$ together with the minimal sets in $N$ which cover $\left.\mathbf{A}\right|_{N}$. This shows that $\left.\mathbf{A}\right|_{M}$ is covered by the minimal sets it contains, contradicting the maximality assumption on $N$.

Corollary 5.6. If $\mathbf{A}$ is a finite solvable algebra with a Maltsev polynomial, then $\mathbf{A}$ is a spread of its type $\mathbf{2}$ minimal sets.

(This establishes for solvable algebras the extra implication (i) $\Rightarrow$ (iii) among the conditions of Theorem 2.12.)

Proof. Theorem 5.4 proves that $\mathbf{A}$ is a spread of its minimal sets. They all have type $\mathbf{2}$, since $\mathbf{A}$ is solvable and has a Maltsev polynomial.

\section{LeFt Nilpotent algebras}

In the papers [3], [5], 6] various forms of nilpotence are discussed. An algebra $\mathbf{A}$ is left nilpotent if $\left[1_{\mathbf{A}}, \ldots,\left[1_{\mathbf{A}},\left[1_{\mathbf{A}}, 1_{\mathbf{A}}\right]\right] \ldots\right]=0_{\mathbf{A}}$ for a sufficiently long expression. This definition implies that the class of left nilpotent algebras is closed under subalgebras and finite direct products. 
Theorem 6.1. A finite left nilpotent algebra has a Maltsev polynomial iff it has a pointed cube polynomial.

(This establishes for nilpotent algebras the extra implication (ii) $\Rightarrow$ (i) among the conditions of Theorem 2.12, )

Proof. A Maltsev polynomial is a 3-ary, 0-pointed, 2-cube polynomial. To prove the converse, we can assume that all elements of $A$ are the interpretations of nullary operation symbols. Thus if $\mathbf{A}$ has a pointed cube polynomial, then it is in fact a pointed cube term, and so it is interpreted as a pointed cube term in every algebra of the variety $\mathcal{V}$ generated by $\mathbf{A}$. Thus, Theorem 2.7 implies that no algebra in $\mathcal{V}$ can have exponential growth rate. However, nontrivial strongly abelian algebras have exponential growth rate by Corollary 2.4. Therefore no such algebra exists in $\mathcal{V}$. We finish the proof by recalling Theorem 6.8 of [9], which states that if a variety $\mathcal{V}$ is generated by a left nilpotent algebra, and there is no nontrivial strongly abelian algebra in $\mathcal{V}$, then $\mathrm{A}$ has a Maltsev polynomial.

The following corollary follows from the fact that if an abelian algebra has a Maltsev polynomial, then it has a Maltsev term.

Corollary 6.2. A finite abelian algebra has a pointed cube polynomial iff it is affine.

Our next goal is to prove the following theorem.

Theorem 6.3. If $\mathbf{A}$ is a finite, left nilpotent algebra, and $\mathbf{A}^{|A|}$ does not have a nontrivial strongly abelian quotient algebra, then $\mathbf{A}$ is a spread of its type $\mathbf{2}$ minimal sets.

(This establishes for nilpotent algebras the extra implication (vi) $\Rightarrow$ (iii) among the conditions of Theorem 2.12.)

Lemma 6.4. Let $\mathbf{A}$ be a finite solvable algebra and $\eta_{1}, \ldots, \eta_{n}$ congruences of $\mathbf{A}$. Then for each type $\mathbf{2}$ covering $\delta \prec \theta$ in $\operatorname{Con}(\mathbf{A})$ such that $\bigwedge_{i=1}^{n} \eta_{i} \leq \delta$ there is an $i$ and congruences $\eta_{i} \leq \alpha \prec \beta$ such that $\langle\delta, \theta\rangle$ and $\langle\alpha, \beta\rangle$ are projective in $\operatorname{Con}(\mathbf{A})$ (and hence have the same minimal sets).

Proof. Let $\gamma$ be an arbitrary congruence of $\mathbf{A}$. We prove that either $\langle\gamma \vee \delta, \gamma \vee \theta\rangle$ or $\langle\gamma \wedge \delta, \gamma \wedge \theta\rangle$ is perspective to $\langle\delta, \theta\rangle$. Indeed, since $\delta \prec \theta$, it is sufficient to prove that $\theta \wedge(\gamma \vee \delta) \neq \theta$ or $\delta \vee(\gamma \wedge \theta) \neq \delta$. Suppose that both inequalities fail. The quotient lattice of $\operatorname{Con}(\mathbf{A})$ modulo the strongly solvability congruence is modular by Theorem 7.7 (4) of [2]. Therefore $\theta=\theta \wedge(\gamma \vee \delta)$ and $\delta=\delta \vee(\gamma \wedge \theta)$ are related by the strongly solvability congruence. This contradicts the fact that $\langle\delta, \theta\rangle$ has type $\mathbf{2}$.

Now apply this observation to $\gamma=\eta_{1}$. Either the statement of the lemma is satisfied with $i=1$, or $\left\langle\eta_{1} \wedge \delta, \eta_{1} \wedge \theta\right\rangle$ is perspective to $\langle\delta, \theta\rangle$. Let $\delta_{1}=\eta_{1} \wedge \delta$ and 
$\theta_{1}$ be any upper cover of $\delta_{1}$ that is below $\eta_{1} \wedge \theta$. Then $\left\langle\delta_{1}, \theta_{1}\right\rangle$ is still perspective to $\langle\delta, \theta\rangle$, since $\delta \vee \theta_{1}=\theta$, but it is now a prime quotient. Perspective prime quotients have the same type, so $\left\langle\delta_{1}, \theta_{1}\right\rangle$ also has type $\mathbf{2}$.

Now apply the previous observation to $\eta_{2}$ and this new quotient. Again, either the statement of the lemma holds for $i=2$, or we can push our cover down below $\eta_{2}$. Continuing this process, if the statement of the lemma fails for every $i$, then we get a prime quotient $\left\langle\delta_{n}, \theta_{n}\right\rangle$ that is still projective to $\langle\delta, \theta\rangle$, and $\delta_{n}=\left(\bigwedge_{i=1}^{n} \eta_{i}\right) \wedge \delta$, while $\theta_{n} \leq\left(\bigwedge_{i=1}^{n} \eta_{i}\right) \wedge \theta$. But our assumption that $\bigwedge_{i=1}^{n} \eta_{i} \leq \delta$ implies that $\theta_{n}=\delta_{n}=$ $\bigwedge_{i=1}^{n} \eta_{i}$, which is a contradiction.

Corollary 6.5. Let $\mathbf{A}$ be a finite solvable algebra and $\mathcal{U}$ a set containing exactly one member from each polynomial isomorphism class of type $\mathbf{2}$ minimal sets of $\mathbf{A}$. If $\delta \prec \theta$ is a covering of type $\mathbf{2}$ in $\mathbf{C o n}\left(\mathbf{A}^{n}\right)$, then there is a $\langle\delta, \theta\rangle$-minimal set of the form $U^{n}$ for some $U \in \mathcal{U}$.

Proof. Denote by $\eta_{1}, \ldots, \eta_{n}$ the projection kernels of $\mathbf{A}^{n}$, and apply Lemma 6.4 to this set of congruences on $\mathbf{A}^{n}$. We get that the covering $\delta \prec \theta$ is projective to a covering $\alpha \prec \beta$ which lies above some $\eta_{i}$. Thus the $\langle\delta, \theta\rangle$-minimal sets are the same as the $\langle\alpha, \beta\rangle$-minimal sets. Identifying $\mathbf{A}$ with $\mathbf{A}^{n} / \eta_{i}$, choose some $U \in \mathcal{U}$ that is an $\left\langle\alpha / \eta_{i}, \beta / \eta_{i}\right\rangle$-minimal set. Then (a) $U^{n}$ is the image of an idempotent unary polynomial of $\mathbf{A}^{n}$, (b) $\left.\mathbf{A}^{n}\right|_{U^{n}}$ is an E-minimal algebra of type $\mathbf{2}$ (since $\left.\mathbf{A}^{n}\right|_{U^{n}}$ is polynomially equivalent to $\left(\left.\mathbf{A}\right|_{U}\right)^{n}$ and powers of solvable E-minimal algebras are E-minimal, according to Lemma 4.10 of [3]), and (c) $\left.\alpha\right|_{U^{n}} \neq\left.\beta\right|_{U^{n}}$. Items (a)-(c) are enough to show that $U^{n}$ is a minimal set for $\langle\alpha, \beta\rangle$ and hence for $\langle\delta, \theta\rangle$.

It is proved in [3] that left nilpotence implies the following, weaker condition:

$$
\mathbf{C}\left(1_{\mathbf{A}}, N^{2} ; \delta\right) \text { holds whenever } \delta \prec \theta \text { and } N \text { is a }\langle\delta, \theta\rangle \text {-trace. }
$$

This condition is clearly still stronger than solvability. One of the main results of [4] is that in an algebra satisfying (†), every maximal subalgebra is a block of a congruence. We shall use this result in the following proof.

Proof of Theorem 6.3. Let $\mathcal{U}=\left\{U_{1}, \ldots, U_{k}\right\}$ be a set containing exactly one member from each polynomial isomorphism class of type 2 minimal sets of $\mathbf{A}$. Let $n=|A|$ and let $\mathbf{B}$ be the subalgebra of $\mathbf{A}^{n}$ generated by all sets of the form $U^{n}$, where $U \in \mathcal{U}$. Thus $B$ is the union of the sets $t^{\mathbf{A}^{n}}\left(U_{1}^{n}, U_{2}^{n}, \ldots, U_{k}^{n}\right)$, where $t$ is a term of $\mathbf{A}$. Clearly $t^{\mathbf{A}^{n}}\left(U_{1}^{n}, U_{2}^{n}, \ldots, U_{k}^{n}\right)=\left(t^{\mathbf{A}}\left(U_{1}, U_{2}, \ldots, U_{k}\right)\right)^{n}$.

There are two cases. If $B=A^{n}$, then let a be a listing of all elements of $\mathbf{A}$. Then $\mathbf{a} \in A^{n}=B$, and so there exists a term $t$ such that $\mathbf{a} \in t^{\mathbf{A}}\left(U_{1}, U_{2}, \ldots, U_{k}\right)^{n}$. Then $t^{\mathbf{A}}\left(U_{1}, U_{2}, \ldots, U_{k}\right)=A$, and so $\mathbf{A}$ is a spread of $\mathcal{U}$.

In the other case, $\mathbf{B}$ is a proper subalgebra of $\mathbf{A}^{n}$. Let $\mathbf{M}$ be a maximal subalgebra of $\mathbf{A}^{n}$ containing $B$. Since $\mathbf{A}^{n}$ is left nilpotent, the result quoted above implies that $M$ is a block of some congruence $\mu$ of $\mathbf{A}^{n}$. We shall prove that $\mathbf{A}^{n} / \mu$ is strongly 
solvable. This is sufficient, since then the quotient modulo any maximal congruence containing $\mu$ is strongly abelian.

Suppose that there is a prime quotient $\mu \leq \delta \prec \theta$ of type 2. Corollary 6.5 states that some $U_{i}^{n}$ is a minimal set for $\langle\delta, \theta\rangle$. This is a contradiction, since $U_{i}^{n} \subseteq B \subseteq M$ is contained in a single $\mu \leq \delta$-class. Thus the proof of Theorem 6.3 is complete.

\section{Abelian varieties}

We show that in an abelian variety, any algebra that is a spread of affine subsets is affine. Note that if $\mathbf{A}$ is a finite abelian algebra, $\langle\alpha, \beta\rangle$ is a prime quotient of type $\mathbf{2}$, and $U \in M_{\mathbf{A}}(\alpha, \beta)$, then the induced algebra $\left.\mathbf{A}\right|_{U}$ is affine. Indeed, $\mathbf{A}$ is solvable, so Lemma 4.27 (4) of [2] implies that the tail of $U$ is empty. By Theorem 4.31 of [2], the induced algebra on $U$ is Maltsev (and E-minimal), and as $\mathbf{A}$ is abelian, it is affine.

Recall that an abelian group operation on an algebra $\mathbf{A}$ is compatible, if adding $x-y+z$ as a term makes $\mathbf{A}$ an affine algebra.

Theorem 7.1. Let $\mathbf{A}$ be an algebra and $U_{1}, \ldots, U_{k} \subseteq A$ such that $A=t\left(U_{1}, \ldots, U_{k}\right)$ for a polynomial $t$ of $\mathbf{A}$. If all induced algebras $\left.\mathbf{A}\right|_{U_{i}}$ are affine, then the following hold.

(1) If $\mathrm{H}\left(\mathbf{A}^{2}\right)$ is abelian, then there is an abelian group operation + on $A$ that is compatible with all operations of $\mathbf{A}$. Moreover, if $\alpha \in \operatorname{Con}(\mathbf{A})$, then $\alpha$ is a congruence of the group $(A,+)$.

(2) If the variety $\mathcal{V}(\mathbf{A})$ generated by $\mathbf{A}$ is abelian, then $\mathbf{A}$ is affine.

(This establishes for algebras generating abelian varieties the extra implication (iii) $\Rightarrow$ (i) among the conditions of Theorem 2.12).

Proof. For each $1 \leq i \leq n$ choose and fix an element $0_{i} \in U_{i}$, a binary polynomial $+_{i}$ and a unary polynomial $-{ }_{i}$ of $\left.\mathbf{A}\right|_{U_{i}}$ such that $\left(U_{i},+_{i},{ }_{-}, 0_{i}\right)$ is an abelian group.

To define the operation + on $A$ let $a, b \in A$. Then $a=t(\mathbf{a})$ and $b=t(\mathbf{b})$ for some $a_{i}, b_{i} \in U_{i}$. Define

$$
a+b=t\left(a_{1}+{ }_{1} b_{1}, \ldots, a_{k}+{ }_{k} b_{k}\right) .
$$

This operation is well-defined, as we now show. Assuming that $a=t\left(\mathbf{a}^{\prime}\right)$, we have to prove that $t\left(a_{1}+{ }_{1} b_{1}, \ldots, a_{k}+{ }_{k} b_{k}\right)=t\left(a_{1}^{\prime}+{ }_{1} b_{1}, \ldots, a_{k}^{\prime}+_{k} b_{k}\right)$. This is an instance of the abelianness of $\mathbf{A}$, because $t(\mathbf{a})=t\left(\mathbf{a}^{\prime}\right)$ implies $t\left(a_{1}+{ }_{1} 0_{1}, \ldots, a_{k}+{ }_{k} 0_{k}\right)=$ $t\left(a_{1}^{\prime}+{ }_{1} 0_{1}, \ldots, a_{k}^{\prime}+{ }_{k} 0_{k}\right)$. The argument is the same for the second variable of + . It is clear that this operation is associative, commutative, $0=t\left(0_{1}, \ldots, 0_{k}\right)$ is a zero element, and $-t(\mathbf{a})=t\left(-{ }_{1} a_{1}, \ldots,-{ }_{k} a_{k}\right)$ is the (well-defined) negative of $t(\mathbf{a})$.

To prove that + is compatible it is enough to show that the congruence $\Delta$ of $\mathbf{A}^{2}$ obtained by collapsing the diagonal satisfies $(a, b) \equiv_{\Delta}(c, d) \Longleftrightarrow a+d=b+c$.

Suppose first that $a+d=b+c$, we prove that $(a, b) \equiv_{\Delta}(c, d)$. Let $a=t(\mathbf{a}), b=t(\mathbf{b})$ and $c=t(\mathbf{c})$. Define $d_{i}=b_{i}+{ }_{i} c_{i}-{ }_{i} a_{i}$, clearly $d=t(\mathbf{d})$. We have $\left(a_{i}, a_{i}\right) \equiv_{\Delta}\left(c_{i}, c_{i}\right)$, 
and using the unary polynomial of $\mathbf{A}^{2}$ that maps $(x, y)$ to $\left(x+{ }_{i} 0_{i}, y+{ }_{i} b_{i}-{ }_{i} a_{i}\right)$ we get that $\left(a_{i}, b_{i}\right) \equiv_{\Delta}\left(c_{i}, d_{i}\right)$. Applying $t$ we see that $(a, b) \equiv_{\Delta}(c, d)$ indeed.

So far, we have only used that $\mathbf{A}$ is abelian, now we need that $\mathbf{A}^{2} / \Delta$ is abelian, too. Suppose that $(a, b) \equiv_{\Delta}(c, d)$. Let $a=t(\mathbf{a}), b=t(\mathbf{b}), c=t(\mathbf{c})$ and $d=t(\mathbf{d})$. We have that

$$
\left(\begin{array}{c}
t\left(a_{1}+{ }_{1} 0_{1}, \ldots, a_{k}+{ }_{k} 0_{k}\right) \\
t\left(b_{1}+{ }_{1} 0_{1}, \ldots, b_{k}+{ }_{k} 0_{k}\right)
\end{array}\right) \equiv_{\Delta}\left(\begin{array}{c}
t\left(c_{1}+{ }_{1} 0_{1}, \ldots, c_{k}+{ }_{k} 0_{k}\right) \\
t\left(d_{1}+{ }_{1} 0_{1}, \ldots, d_{k}+{ }_{k} 0_{k}\right)
\end{array}\right) .
$$

Since $\mathbf{A}^{2} / \Delta$ is abelian, we can replace each $0_{i}$ in the second row by $a_{i}-{ }_{i} b_{i}$. Therefore

$$
\left(\begin{array}{l}
t\left(a_{1}, \ldots, a_{k}\right) \\
t\left(a_{1}, \ldots, a_{k}\right)
\end{array}\right) \equiv_{\Delta}\left(\begin{array}{c}
t\left(c_{1}, \ldots, c_{k}\right) \\
t\left(d_{1}+{ }_{1} a_{1}-{ }_{k} b_{1}, \ldots, d_{k}+{ }_{k} a_{k}-{ }_{k} b_{k}\right)
\end{array}\right) .
$$

That is, $(a, a) \equiv_{\Delta}(c, d+a-b)$. The abelianness of $\mathbf{A}$ implies that the diagonal is a $\Delta$-class, hence $c=d+a-b$. Thus + is indeed a compatible operation.

To prove that every congruence $\alpha$ of $\mathbf{A}$ is a group-congruence it is sufficient to verify that if $(a, b) \in \alpha$, then $(a+c, b+c) \in \alpha$. Again, let $a=t(\mathbf{a}), b=t(\mathbf{b})$ and $c=t(\mathbf{c})$. Then

$$
t\left(a_{1}+{ }_{1} 0_{1}, \ldots, a_{k}+{ }_{k} 0_{k}\right) \equiv_{\alpha} t\left(b_{1}+{ }_{1} 0_{1}, \ldots, b_{k}+{ }_{k} 0_{k}\right) .
$$

Since $\mathbf{A} / \alpha$ is abelian, we can move each $0_{i}$ to $c_{i}$, proving (1).

Now suppose that $\mathcal{V}(\mathbf{A})$ is abelian. Recall that an algebra is called Hamiltonian, if every subalgebra is a block of some congruence. By the main result of [11], every member of $\mathcal{V}(\mathbf{A})$ is Hamiltonian. Notice that $A^{n}=\hat{t}\left(U_{1}^{n}, \ldots, U_{k}^{n}\right)$ holds in $\mathbf{A}^{n}$, where $\hat{t}$ is $t$ acting componentwise. Hence (1) applies to all powers of $\mathbf{A}$, and the compatible group operation on $\mathbf{A}^{n}$ is the operation of the group $(A,+)^{n}$. Construct the free algebra $\mathbf{F}$ of $\mathcal{V}(\mathbf{A})$ generated by $x, y$ and $z$ as a subalgebra of $\mathbf{A}^{n}$, where $n=|A|^{3}$. By the Hamiltonian property, there exists a congruence $\alpha$ of $\mathbf{A}^{n}$ such that $F$ is a class of $\alpha$. Item (1) of the theorem implies that $\alpha$ is a group congruence, so $F$ is a coset modulo a subgroup, hence $x-y+z \in F$. Therefore $x-y+z$ is a term operation of $\mathbf{A}$.

We present two examples showing that no obvious weakening of the conditions in the previous theorem is possible. Our starting point is the example following Corollary 4.4 of [10].

Example 7.2. Let $\mathbb{Z}_{2}$ be the two-element field, let $A=\mathbb{Z}_{2}^{3}$, denote by + the (elementary abelian) group operation on $A$, and consider the following matrices in $\mathbb{Z}_{2}^{3 \times 3}$ :

$$
F_{1}=\left(\begin{array}{lll}
1 & 0 & 0 \\
0 & 1 & 0 \\
1 & 0 & 0
\end{array}\right), \quad F_{2}=\left(\begin{array}{ccc}
1 & 0 & 0 \\
0 & 1 & 0 \\
0 & 0 & 0
\end{array}\right), \quad G=\left(\begin{array}{lll}
1 & 0 & 0 \\
1 & 1 & 0 \\
0 & 0 & 1
\end{array}\right)
$$


Define a binary operation $*$ on $A$ by $u * v=F_{1} u+F_{2} v$ (matrix-vector multiplication) and a unary operation $g$ on $A$ by $g(v)=G v+(1,0,0)^{T}$, so

$$
g:\left(\begin{array}{l}
a \\
b \\
c
\end{array}\right) \mapsto\left(\begin{array}{c}
a+1 \\
a+b \\
c
\end{array}\right) .
$$

These are affine operations, so the algebra $\mathbf{A}=\langle A ; *, g\rangle$ is abelian, and $x-y+z$ is a compatible operation. Define three subgroups of $\langle A ;+\rangle$ as follows: $B$ is the subgroup generated by $(0,0,1)^{T}, C$ is the subgroup generated by $(0,0,1)^{T}$ and $(0,1,0)^{T}$, finally $D$ is the subgroup generated by $(0,1,0)^{T}$. Let $\beta, \gamma$ and $\delta$ denote the corresponding congruences of $\langle A ;+\rangle$. It is easy to check by hand or by computer that $\mathbf{A}$ has only these three nontrivial proper congruences. We have $\beta \wedge \delta=0_{A}$ and $\beta \vee \delta=\gamma$. It can be verified also that $\mathrm{H}(\mathbf{A})$ is abelian.

The type of $\left\langle 0_{A}, \beta\right\rangle$ is $\mathbf{1}$, while both $\langle\beta, \gamma\rangle$ and $\left\langle\gamma, 1_{A}\right\rangle$ have type $\mathbf{2}$. These latter quotients have the same minimal sets. There are four such type $\mathbf{2}$ minimal sets, one of which is $U=\left\{(0,0,0)^{T},(0,1,0)^{T},(1,0,0)^{T},(1,1,0)^{T}\right\}$. Thus the induced algebra $\left.\mathbf{A}\right|_{U}$ is affine (since $\mathbf{A}$ is abelian). We also have that $U * U=A$, so the initial condition in Theorem 7.1 is satisfied.

The algebra $\mathbf{A}^{2} / \Delta$ is an 8-element abelian algebra (although it has a nonabelian quotient, so $\mathbf{H}\left(\mathbf{A}^{2}\right)$ is not abelian). Nevertheless, the abelianness of $\mathbf{A}^{2} / \Delta$ and $\mathbf{H}(\mathbf{A})$ are sufficient to make the proof of statement (1) of Theorem 7.1 work for $\mathbf{A}$ (which indeed has a compatible + operation). But $\mathbf{A}$ is not affine, so we cannot drop the assumption from statement (2) in Theorem 7.1 that $\mathcal{V}(\mathbf{A})$ is abelian.

Example 7.3. Now let $A, *, g$ be as in the preceding example, and let $h$ be the transposition of $A$ which switches $(1,0,0)^{T}$ and $(1,0,1)^{T}$ (and fixes all other elements of $A)$. Let $\overline{\mathbf{A}}=\langle A ; *, g, h\rangle$. This algebra has only four congruences, the nontrivial proper ones are $\beta$ and $\gamma$ above. The quotients $\langle\beta, \gamma\rangle$ and $\left\langle\gamma, 1_{A}\right\rangle$ still have type $\mathbf{2}$, and they have the same minimal sets, but now there are 16 of them. One of these is $U$ above, so the initial condition in Theorem 7.1 is satisfied again. It is also true that $\mathbf{H}(\overline{\mathbf{A}})$ is abelian. However, $h$ is not linear, and $\overline{\mathbf{A}}^{2} / \Delta$ is a 5-element nonabelian algebra. Therefore it is not sufficient to assume in (1) of Theorem 7.1 that $H(\mathbf{A})$ is abelian.

\section{Semisimple algebras}

An algebra is called semisimple if it is isomorphic to a subdirect product of simple algebras, or equivalently, if its maximal congruences intersect to zero.

Theorem 8.1. Let $\mathbf{A}$ be a finite, semisimple, solvable algebra. If the growth rate of $\mathbf{A}$ is linear, then $\mathbf{A}$ has a Maltsev polynomial, and so it is affine. 
(This establishes for semisimple abelian algebras the extra implication (iv) $\Rightarrow$ (i) among the conditions of Theorem 2.12)

We shall need the following corollary of Lemma 6.4.

Corollary 8.2. Let $\mathbf{A}$ be a finite solvable algebra, $\eta_{1}, \ldots, \eta_{n}$ maximal congruences of $\mathbf{A}$ and $\eta=\bigwedge_{i=1}^{n} \eta_{i}$. Suppose that

(1) For each $1 \leq i \leq n$, the $\left\langle\eta_{i}, 1\right\rangle$-minimal sets are the same (that is, $M_{\mathbf{A}}\left(\eta_{i}, 1\right)=$ $M_{\mathbf{A}}\left(\eta_{j}, 1\right)$ for every $i$ and $\left.j\right)$.

(2) $A / \eta$ has no nontrivial strongly abelian quotient algebras.

Then $\langle\eta, 1\rangle$ is a tame quotient, and if $\theta \geq \eta$ is a coatom in $\operatorname{Con}(\mathbf{A})$, then $M_{\mathbf{A}}(\theta, 1)=$ $M_{\mathbf{A}}\left(\eta_{1}, 1\right)$.

Proof. Clearly, for every upper cover $\delta$ of $\eta$, the quotient $\langle\eta, \delta\rangle$ is perspective to some $\left\langle\eta_{i}, 1\right\rangle$ (and so has type 2). Condition (2) implies that for every maximal congruence $\theta \geq \eta$ the quotient $\langle\theta, 1\rangle$ also has type $\mathbf{2}$. Therefore it is projective to some $\left\langle\eta_{i}, 1\right\rangle$ by Lemma 6.4. Thus, if $U$ is an $\left\langle\eta_{1}, 1\right\rangle$-minimal set, then it is minimal with respect to each such quotient $\langle\eta, \delta\rangle$ and $\langle\theta, 1\rangle$, and also $U \in M_{\mathbf{A}}(\eta, 1)$. This implies that restriction to $U$ is a 0,1 -separating map, so by Definition 2.6 of [2], the quotient $\langle\eta, 1\rangle$ is tame.

Proof of Theorem 8.1. Our assumption that the growth rate of $\mathbf{A}$ is linear implies, by Corollary 2.4 (1), that

$$
\text { no power of } \mathbf{A}^{n} \text { of has a nontrivial strongly abelian homomorphic image. }
$$

In particular, if $\alpha_{1}, \ldots, \alpha_{\ell}$ is the list of all maximal congruences of $\mathbf{A}$, then for each $i$, the solvability of $\mathbf{A}$ implies that $\mathbf{A} / \alpha_{i}$ is abelian, and (8.1) implies that it is not strongly abelian. Therefore, $\left\langle\alpha_{i}, 1\right\rangle$ has type $\mathbf{2}$ for each $i$. It follows also that $\mathbf{A}$ is abelian, since it is a subdirect product of the abelian algebras $\mathbf{A} / \alpha_{i}$.

Call two maximal congruences $\alpha_{i}$ and $\alpha_{j}$ of $\mathbf{A}$ equivalent, if the $\left\langle\alpha_{i}, 1\right\rangle$-minimal sets and the $\left\langle\alpha_{j}, 1\right\rangle$-minimal sets are the same. This is an equivalence relation. For each equivalence class, consider the intersection $\beta_{i}$ of its members. Thus we get congruences $\beta_{1}, \ldots, \beta_{m}$ of $\mathbf{A}$ such that their intersection is zero. In view of (8.1), Corollary 8.2 shows that $\left\langle\beta_{i}, 1\right\rangle$ is a tame quotient for every $i$, and the set of all coatoms above $\beta_{i}$ forms an equivalence class, for every $i$. Denote $\mathbf{A} / \beta_{i}$ by $\mathbf{B}_{i}$. Then A can be viewed as a subdirect subalgebra of $\mathbf{B}_{1} \times \cdots \times \mathbf{B}_{m}$.

Fix a minimal set $U_{i}$ corresponding to the coatoms whose intersection is $\beta_{i}$. This is a minimal set for the tame quotient $\left\langle\beta_{i}, 1\right\rangle$. Since $\mathbf{A}$ is abelian, its type $\mathbf{2}$ minimal sets are affine and E-minimal (see the remark at the beginning of Section 7). This means that if $\alpha_{i}$ and $\alpha_{j}$ are not equivalent, then $\alpha_{i}$ collapses every $\left\langle\alpha_{j}, 1\right\rangle$-minimal set (by which we mean that every $\left\langle\alpha_{j}, 1\right\rangle$-minimal set is contained in a single $\alpha_{i}$-class). Therefore if $i \neq j$, then $\beta_{i}$ collapses $U_{j}$. Hence $\beta_{i}$ restricts trivially to $U_{i}$ (that is, 
$\left.\left.\beta_{i}\right|_{U_{i}}=0_{U_{i}}\right)$, since $\beta_{1} \wedge \ldots \wedge \beta_{m}=0$. In fact, $U_{i}$ is a $\left\langle\beta_{i}, 1\right\rangle$-trace, and therefore $U_{i} / \beta_{i}$ is polynomially isomorphic to a vector space over a finite field.

Since $\mathbf{A}$ is abelian and satisfies (8.1), Theorem 6.3 implies that $\mathbf{A}$ is a spread of its type $\mathbf{2}$ minimal sets. Applying Lemma 6.4 to $\mathbf{A}$ and its maximal congruences $\alpha_{1}, \ldots, \alpha_{\ell}$ we see that every type $\mathbf{2}$ quotient of $\mathbf{A}$ has the same minimal sets as $\left\langle\alpha_{i}, 1\right\rangle$ for some $\alpha_{i}$. In a spread, each set can be replaced with a polynomially isomorphic one, therefore we can use the representatives $U_{1}, \ldots, U_{m}$. Thus there exists a polynomial $t$ such that

$$
t\left(U_{1}, \ldots, U_{1}, U_{2}, \ldots, U_{2}, \ldots, U_{m}, \ldots, U_{m}\right)=A .
$$

Write this polynomial as $t\left(\mathbf{x}^{1}, \mathbf{x}^{2}, \ldots, \mathbf{x}^{m}\right)$, where $\mathbf{x}^{i}$ is the string of variables where $U_{i}$ occurs.

Let $0_{i} \in U_{i}$ be fixed arbitrarily, and substitute $0_{i}$ to every variable in $\mathbf{x}^{i}$ for $i \neq 1$. We get a polynomial

$$
f\left(\mathbf{x}^{1}\right)=f\left(x_{1}^{1}, \ldots, x_{n}^{1}\right)=t\left(\mathbf{x}^{1}, \hat{0}_{2}, \ldots, \hat{0}_{m}\right)
$$

of A. Let $T_{1}=f\left(U_{1}, \ldots, U_{1}\right)$.

Each $\beta_{j}$ collapses $T_{1}$ whenever $j \neq 1$, and therefore $\beta_{1}$ restricts trivially to $T_{1}$ (using again that $\beta_{1} \wedge \ldots \wedge \beta_{m}=0$ ). Therefore, as $U_{1}$ is a $\left\langle\beta_{1}, 1\right\rangle$-trace, for every $a \neq b \in T_{1}$ there exists a unary polynomial mapping $T_{1}$ to $U_{1}$ that separates $a$ and $b$. Thus we can apply Lemma 3.8 of [9] to $S=U_{1}$, zero element $0_{1}$ and $f$. The proof of this lemma shows that there exist

(1) unary polynomials $g_{1}, \ldots, g_{k}$ mapping $T_{1}$ to $U_{1}$, that satisfy $g_{i}\left(0_{1}\right)=0_{1}$, and

(2) $k$-ary polynomials $\ell_{1}, \ldots, \ell_{n}$ satisfying $\ell_{i}\left(\hat{0}_{1}\right)=0_{1}$ and $\ell_{i}\left(U_{1}, \ldots, U_{1}\right) \subseteq U_{1}$

such that for $f^{\prime}(\mathbf{y})=f\left(\ell_{1}(\mathbf{y}), \ldots, \ell_{n}(\mathbf{y})\right)$ we have

$$
g_{i}\left(f^{\prime}\left(y_{1}, \ldots, y_{k}\right)\right)=y_{i}
$$

when each $y_{1}, \ldots, y_{k} \in U_{1}$ and

$$
f^{\prime}\left(g_{1}(x), \ldots, g_{k}(x)\right)=x
$$

for every $x \in T_{1}$. Thus $f^{\prime}\left(U_{1}, \ldots, U_{1}\right)=T_{1}$. Note that $f^{\prime}\left(\hat{0}_{1}\right)=f\left(\hat{0}_{1}\right)$ holds.

We defined polynomials $g_{i}=g_{i}^{1}$ and $\ell_{i}=\ell_{i}^{1}$ above for the first block of variables $\mathbf{x}^{1}$ of the polynomial $t\left(\mathbf{x}^{1}, \ldots, \mathbf{x}^{m}\right)$. Do this in an analogous way for all other blocks of variables $\mathbf{x}^{j}$ to obtain unary polynomials $g_{i}^{j}\left(1 \leq i \leq k_{j}\right)$ and $k_{j}$-ary polynomials $\ell_{i}^{j}$ $\left(1 \leq i \leq n_{j}\right)$, and the set $T_{j}$. We shall substitute these polynomials into $t$ in the following way. Consider the $j$-th block $\mathbf{x}^{j}=\left(x_{1}^{j}, \ldots, x_{n_{j}}^{j}\right)$ of the variables of $t$. Then make the substitution

$$
x_{i}^{j} \rightarrow \ell_{i}^{j}\left(g_{1}^{j}\left(y_{j}\right), \ldots, g_{k_{j}}^{j}\left(y_{j}\right)\right)
$$

for $1 \leq i \leq n_{j}$ and $1 \leq j \leq m$. This way, we get a polynomial $r\left(y_{1}, \ldots, y_{m}\right)$. 
Equation (8.3) and its analogues ensure that

$$
r\left(0_{1}, \ldots, 0_{j-1}, c, 0_{j+1}, \ldots, 0_{m}\right)=c
$$

holds whenever $c \in T_{j}$. Thus if $c_{j} \in T_{j}$, then $r\left(c_{1}, \ldots, c_{m}\right) \equiv_{\beta_{j}} c_{j}$.

Let $a \in A$. By equation (8.2) we have $a=t\left(\mathbf{a}^{1}, \ldots, \mathbf{a}^{m}\right)$ for appropriate vectors $\mathbf{a}^{j}$ such that each component of $\mathbf{a}^{j}$ is in $U_{j}$. Replace each $\mathbf{a}^{j}$ by $\hat{0}_{j}$ for $j \geq 2$, and call the resulting element $c_{1} \in T_{1}$. Since each $U_{j}$ is contained in a $\beta_{i}$-class for every $i \neq j$ we see that $c_{1} \equiv_{\beta_{1}} a$. Do the analogous substitutions for all other variables to obtain elements $c_{i} \in T_{i}$ such that $a \equiv_{\beta_{i}} c_{i}$. Then $r\left(c_{1}, \ldots, c_{m}\right) \equiv_{\beta_{i}} a$ for every $i$, and since the $\beta_{i}$-s intersect to zero we have that $r\left(c_{1}, \ldots, c_{m}\right)=a$.

We show that if $c_{j}^{\prime} \in T_{j}$ are such that $r\left(c_{1}^{\prime}, \ldots, c_{m}^{\prime}\right)=a$, then $c_{j}=c_{j}^{\prime}$ for every $j$. Indeed, $c_{j} \equiv_{\beta_{j}} a=r\left(c_{1}^{\prime}, \ldots, c_{m}^{\prime}\right) \equiv_{\beta_{j}} c_{j}^{\prime}$, and $\beta_{j}$ restricts trivially to $T_{j}$.

In other words, $r: T_{1} \times \cdots \times T_{m} \rightarrow A$ is a bijection. Corollary 3.7 of [9] states that $T_{j}$ is the range of an idempotent polynomial $e_{j}$, and the induced algebra on $T_{j}$ is isomorphic to an algebra polynomially equivalent to a full matrix power of $\left.\mathbf{A}\right|_{U_{j}}$. Therefore $T_{j}$ has an induced Maltsev polynomial $d_{j}$. Let

$$
d(x, y, z)=r\left(\ldots, d_{j}\left(e_{j}(x), e_{j}(y), e_{j}(z)\right), \ldots\right) .
$$

We prove that $d(x, x, z)=r\left(\ldots, e_{j}(z), \ldots\right)=z$. Let $z=r\left(z_{1}, \ldots, z_{m}\right)$, where $z_{j} \in T_{j}$. Then $z \equiv_{\beta_{j}} z_{j}$, so $e_{j}(z) \equiv_{\beta_{j}} e_{j}\left(z_{j}\right)=z_{j}$, and so $e_{j}(z)=z_{j}$, since $\beta_{j}$ restricts trivially to $T_{j}$. Similarly, $d(x, z, z)=x$. Hence $d$ is a Maltsev polynomial of $\mathbf{A}$ and the proof of Theorem 8.1 is complete.

We actually proved that $\mathbf{A}$ is the full direct product of the algebras $\mathbf{B}_{i}$, since $T_{i}$ and $B_{i}$ are in a bijective correspondence via factoring modulo $\beta_{i}$.

The following example shows that the direct product of two affine algebras need not have a Maltsev polynomial, even if its growth rate is linear, and so Theorem 8.1 cannot be improved to say that if $\mathbf{A} / \alpha_{1}$ and $\mathbf{A} / \alpha_{2}$ have Maltsev polynomials, then so does $\mathbf{A} /\left(\alpha_{1} \wedge \alpha_{2}\right)$.

Example 8.3. Consider $V=\mathbb{Z}_{2}^{2}$ as a vector space over the two-element field $\mathbb{Z}_{2}^{2}$. Let

$$
P=\left(\begin{array}{ll}
1 & 0 \\
0 & 0
\end{array}\right) \quad \text { and } \quad N=\left(\begin{array}{ll}
0 & 0 \\
1 & 0
\end{array}\right) \text {. }
$$

Define an operation $f$ on $V$ by

$$
f(\mathbf{x}, \mathbf{y})=P \mathbf{x}+N \mathbf{y} .
$$

Clearly, $P^{2}=P, P N=N^{2}=0$ and $N P=N$. Therefore $\{0, P, N\}$ is a semigroup under matrix multiplication, and the clone of all term operations of the algebra $\langle V ;+, f, \mathbf{0}\rangle$ consists of all functions of the form

$$
M_{1} \mathbf{x}_{1}+\ldots+M_{n} \mathbf{x}_{n}
$$


where each $M_{i}$ is either $P, N$, or the identity matrix. If one deletes + from the basic operations, then the identity matrix cannot occur as a coefficient (except for projections), and there can be at most one instance of $P$ and at most one instance of $N$. It is left to the reader to check that $f(V, V)=V$.

Next we define two algebras $\mathbf{B}$ and $\mathbf{C}$. The language contains the operation symbols ,$+ \oplus$, a 4 -ary $g$, and 0 . In the algebra $\mathbf{B}$ the underlying set is $V,+$ interprets as the usual addition, $\oplus$ as the constant zero function, and 0 as the zero vector $\mathbf{0}$. In the algebra $\mathbf{C}$, the only difference is that + is interpreted as constant zero, and $\oplus$ as the usual addition of $V$. Finally,

$$
\begin{aligned}
& g^{\mathbf{B}}(\mathbf{x}, \mathbf{y}, \mathbf{u}, \mathbf{v})=P \mathbf{x}+N \mathbf{y}=f(\mathbf{x}, \mathbf{y}) \\
& g^{\mathbf{C}}(\mathbf{x}, \mathbf{y}, \mathbf{u}, \mathbf{v})=P \mathbf{u} \oplus N \mathbf{v}=f(\mathbf{u}, \mathbf{v})
\end{aligned}
$$

Both algebras $\mathbf{B}$ and $\mathbf{C}$ have Maltsev terms, namely $x-y+z$ for $\mathbf{B}$ and $x \ominus y \oplus z$ for $\mathbf{C}$. In addition, both algebras are abelian, hence affine. Now consider the algebra $\mathbf{A}=\mathbf{B} \times \mathbf{C}$. It follows that $\mathbf{A}$ is also abelian. Our goal is to show that $\mathbf{A}$ has linear growth rate, but has no Maltsev polynomial.

To see that the growth rate of $\mathbf{A}$ is linear, observe first that $e_{B}(x)=x+(\mathbf{0}, \mathbf{0})$ is an idempotent unary polynomial of A mapping $A=B \times C$ to the subset $U=B \times\{\mathbf{0}\}$. Similarly, $e_{C}(x)=x \oplus(\mathbf{0}, \mathbf{0})$ is an idempotent unary polynomial of $\mathbf{A}$ mapping $A$ to the subset $W=\{\mathbf{0}\} \times C$. The induced algebras $\left.\mathbf{A}\right|_{U}$ and $\left.\mathbf{A}\right|_{W}$ are abelian and have Maltsev polynomials, hence they are affine algebras. Moreover,

$$
g^{\mathbf{A}}(U, U, W, W)=A
$$

Thus A has linear growth rate by Theorem 2.5, Lemma 5.2 and Corollary 2.4 (2).

It remains to prove that $\mathbf{A}$ does not have a Maltsev polynomial. Suppose that $\mathbf{A}$ has a Maltsev polynomial. Since $\mathbf{A}$ is abelian, we get that $\mathbf{A}$ is affine, and hence it has a Maltsev term $M(x, y, z)$. Since $\mathbf{B}, \mathbf{C}$ are affine, each one has a unique Maltsev term, so $M^{\mathbf{B}}(x, y, z)=x-y+z$ and $M^{\mathbf{C}}(x, y, z)=x \ominus y \oplus z$. Thus $M^{\mathbf{A}}$ acts componentwise as $x-y+z$ in the $\mathbf{B}$-coordinate and as $x \ominus y \oplus z$ in the $\mathbf{C}$-coordinate. In particular, if $\mathbf{v}=(0,1)^{T} \in V$, then for the elements $(\mathbf{v}, \mathbf{0}),(\mathbf{0}, \mathbf{0}),(\mathbf{0}, \mathbf{v})$ of $A$ we get that $M^{\mathbf{A}}((\mathbf{v}, \mathbf{0}),(\mathbf{0}, \mathbf{0}),(\mathbf{0}, \mathbf{v}))=(\mathbf{v}, \mathbf{v})$. This implies that $\{(\mathbf{v}, \mathbf{0}),(\mathbf{0}, \mathbf{0}),(\mathbf{0}, \mathbf{v})\}$ is not a subuniverse of $\mathbf{A}$. On the other hand, it is not hard to check that the set $\{(\mathbf{v}, \mathbf{0}),(\mathbf{0}, \mathbf{0}),(\mathbf{0}, \mathbf{v})\}$ is closed under all operations $+, \oplus, g, 0$ of $\mathbf{A}$. Indeed, closure under + and $\oplus$ follows, because + and $\oplus$ are constant $\mathbf{0}$ in one of the components and the usual addition of $V$ in the other, and $\{\mathbf{v}, \mathbf{0}\}$ is closed under addition. Finally, closure under $g$ follows from the fact that we have $f(\mathbf{v}, \mathbf{v})=f(\mathbf{v}, \mathbf{0})=f(\mathbf{0}, \mathbf{v})=\mathbf{0}$, since $P \mathbf{v}=N \mathbf{v}=\mathbf{0}$. This shows that $\{(\mathbf{v}, \mathbf{0}),(\mathbf{0}, \mathbf{0}),(\mathbf{0}, \mathbf{v})\}$ is a subuniverse of $\mathbf{A}$. The contradiction obtained proves that $\mathbf{A}$ has no Maltsev polynomial. 
In this example, $\mathbf{A}$ is abelian, $d_{\mathbf{A}}(n) \in O(n)$, and yet $\mathbf{A}$ is not affine. This proves that, for abelian algebras, no one of the equivalent conditions (iii), (iv), (v) or (vi) implies any one of the equivalent conditions (i) or (ii).

\section{Summary of Results. Problems.}

We are now in a position to prove Theorem 2.12, which summarizes our main results.

Proof of Theorem 2.12. [(i) $\Rightarrow$ (iv)] A Maltsev polynomial is a 3-ary, 0-pointed, 2-cube term. Hence this implication follows from Corollary 2.8 which implies that, if $\mathbf{A}$ is an algebra with a 0 -pointed, $k$-cube polynomial, and $\mathbf{A}^{k}$ is finitely generated, then $d_{\mathbf{A}}(n) \in O\left(n^{k-1}\right)$.

$[(\mathrm{i}) \Rightarrow(\mathrm{ii})]$ The definition of a pointed cube polynomial generalizes that of a Maltsev polynomial.

$[(\mathrm{ii}) \Rightarrow(\mathrm{v})]$ Theorem 2.7 shows that if $\mathbf{A}$ is an algebra with a $p$-pointed $k$-cube polynomial, and $p \geq 1$, then $d_{\mathbf{A}}(n)$ is bounded above by a polynomial in $n$ if $\mathbf{A}^{p+k-1}$ is finitely generated. The restriction on $\mathbf{A}^{p+k-1}$ is satisfied when $\mathbf{A}$ is finite. This proves that (ii) implies (v) when $p \geq 1$. The case $p=0$ is handled similarly using Corollary 2.8.

$[($ iii $) \Rightarrow($ iv $)]$ A binary polynomial with a unit element is a 2-ary, 1-pointed, 2-cube polynomial. Corollary 2.8 implies that the growth rate of any finite algebra that has such a polynomial lies in $O(n)$. The induced algebra on an $\langle\alpha, \beta\rangle$-minimal set of type $\mathbf{2}, \mathbf{3}, \mathbf{4}$ or $\mathbf{5}$ has such a binary polynomial: take $d(x, 0, y)$ with $d$ a pseudo-Maltsev polynomial and 0 in the body if the type is 2 , and take $x \wedge y$ with $\wedge$ a pseudo-meet polynomial in the other cases. Therefore, by Lemma 5.2 of this paper, $d_{\mathbf{A}}(n) \in O(n)$ whenever $\mathbf{A}$ is a spread of minimal sets whose types are not $\mathbf{1}$.

$[(\mathrm{iv}) \Rightarrow(\mathrm{v})] O(n) \cap 2^{\Omega(n)}=\emptyset$.

$[(\mathrm{v}) \Rightarrow(\mathrm{vi})]$ Theorem 2.2 implies that $d_{\mathbf{A}}(n) \notin 2^{\Theta(n)}$ is equivalent to $d_{\mathbf{A}}(n) \notin 2^{\Omega(n)}$ for finite algebras. Hence $(\mathrm{v}) \Rightarrow(\mathrm{vi})$ is just the contrapositive of Corollary 2.4 (1).

$[(\mathrm{vi}) \nRightarrow(\mathrm{v})]$ Example 5.3.5 of [7] describes finite implication algebras with exponential growth. These satisfy (vi), since no nontrivial implication algebra is strongly abelian, but do not satisfy (v).

$[(\mathrm{ii}) \not \Rightarrow(\mathrm{iv}),(\mathrm{v}) \nRightarrow(\mathrm{iv})]$ Given $k \geq 2$, Theorem 5.3.1 of [7] constructs a finite algebra with a cube polynomial whose $d$-function satisfies $d_{\mathbf{A}}(n) \in \Theta\left(n^{k-1}\right)$. When $k=3$ one has $d_{\mathbf{A}}(n) \notin 2^{\Omega(n)}$, yet $d_{\mathbf{A}}(n) \notin O(n)$.

$\left[(\mathrm{i}) \not \nexists(\right.$ iii $),($ iv $) \neq$ (iii)] If $\mathbf{A}$ is a 2-element Boolean algebra, then $d_{\mathbf{A}}(n) \in O(\log (n))$ (hence $d_{\mathbf{A}}(n) \in O(n)$ ), and therefore (i) and (iv) hold. But (iii) does not hold, since A has no type $\mathbf{2}$ minimal sets.

$[($ iii $) \not$ (ii)] Example 8.3 describes an abelian algebra $\mathbf{C}$ that is a spread of type $\mathbf{2}$ minimal sets, but does not have a Maltsev term. If $\mathbf{C}$ had a pointed cube polynomial, then by Theorem 6.1 it would have Maltsev polynomial. But it is well known that 
an abelian algebra with a Maltsev polynomial has a Maltsev term, and $\mathbf{C}$ does not have such a term.

[(iv) $\nRightarrow$ (ii)] According to Theorem 2.11, any function that can be realized as the growth rate of a finite algebra can also be realized as the growth rate of a finite algebra that does not have a pointed cube polynomial.

Recall the six growth-restricting conditions portrayed in this theorem:

(i) A has a Maltsev polynomial.

(ii) A has a pointed cube polynomial.

(iii) $\mathbf{A}$ is a spread of its type $\mathbf{2}$ minimal sets.

(iv) $d_{\mathbf{A}}(n) \in O(n)$.

(v) $d_{\mathbf{A}}(n) \notin 2^{\Omega(n)}$.

(vi) No finite power $\mathbf{A}^{n}$ has a nontrivial strongly abelian homomorphic image.

We have shown that for arbitrary finite algebras, the following implications hold:

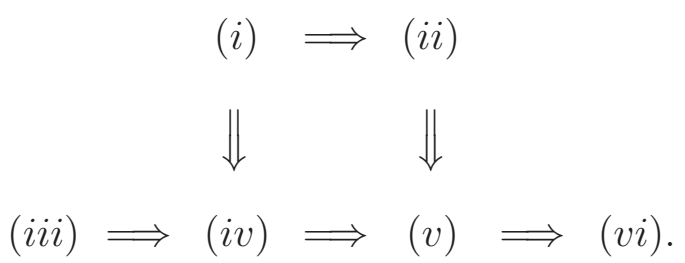

If $\mathbf{A}$ is a finite solvable algebra, then this can be strengthened to

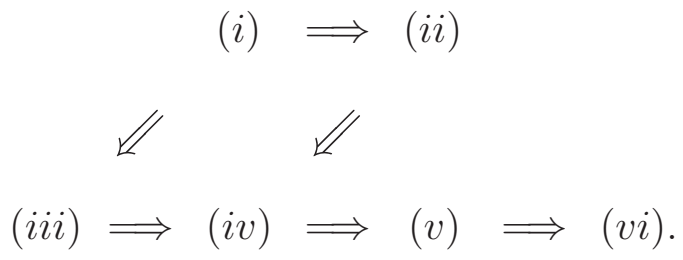

If $\mathbf{A}$ is a finite left nilpotent algebra, then we have established that

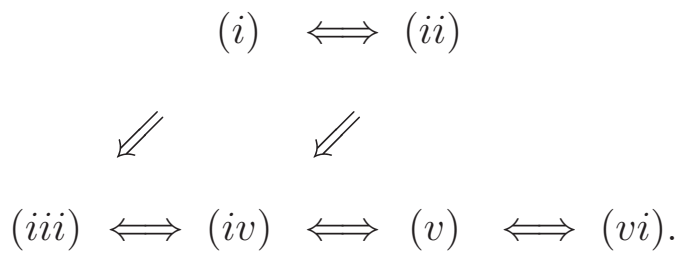

Finally, if $\mathbf{A}$ is a semisimple abelian algebra or if it generates an abelian variety, then all six conditions are equivalent. On the other hand, we gave an example of an abelian algebra satisfying the properties in the bottom row but not satisfying those in the top row, so no other implications hold for finite abelian or nilpotent algebras.

Now let us return to the "solvability" diagram. The example preceding Theorem 6.10 in [9], which is a finite, solvable algebra which has no Maltsev polynomial, but has a binary polynomial with a unit element, shows that (ii) $\nRightarrow$ (i) for finite solvable algebras. We have seen that no item on the bottom row implies any item on the 
top row for solvable algebras, so the true implications yet to be discovered can only be (ii) $\Rightarrow$ (iii), or the reversal of some of the implications along the bottom row. This suggests some problems.

Problem 9.1. Does (ii) $\Rightarrow$ (iii) hold for finite solvable algebras?

Problem 9.2. Which of the true implications (iii) $\Rightarrow(\mathrm{iv}) \Rightarrow(\mathrm{v}) \Rightarrow(\mathrm{vi})$ can be reversed for finite solvable algebras?

\section{REFERENCES}

[1] Berman, Joel, McKenzie, Ralph, Clones satisfying the term condition. Discrete Math. 52 (1984), 7-29.

[2] Hobby, David, McKenzie, Ralph, The structure of finite algebras. Contemporary Mathematics, 76. American Mathematical Society, Providence, RI, 1988.

[3] Kearnes, Keith, An order-theoretic property of the commutator. Internat. J. Algebra Comput. 3 (1993), 491-533.

[4] Kearnes, Keith, A Hamiltonian property for nilpotent algebras. Algebra Universalis 37 (1997), 403-421.

[5] Kearnes, Keith, Kiss, Emil, Finite algebras of finite complexity. Discrete Math. 207 (1999), $89-135$.

[6] Kearnes, Keith, Kiss, Emil, Residual smallness and weak centrality. International Journal of Algebra and Computation 13 (2003), 35-59.

[7] Kearnes, Keith, Kiss, Emil, Szendrei, Ágnes, Growth rates of algebras I: pointed cube terms. manuscript.

[8] Kearnes, Keith, Kiss, Emil, Szendrei, Ágnes, Growth rates of algebras II: Wiegold dichotomy. manuscript.

[9] Kearnes, Keith A., Kiss, Emil W., Valeriote, Matthew A., Minimal sets and varieties. Trans. Amer. Math. Soc. 350 (1998), 1-41.

[10] Kearnes, Keith A., Kiss, Emil W., Valeriote, Matthew A., A geometric consequence of residual smallness. Annals of Pure and Applied Logic 99 (1999), 137-169.

[11] Kiss, Emil W., Valeriote, Matthew A., Abelian algebras and the Hamiltonian property. J. Pure Appl. Algebra 87 (1993), no. 1, 37-49.

[12] Quick, Martyn, Ruškuc, Nik, Growth of generating sets for direct powers of classical algebraic structures. J. Austral. Math. Soc. 89 (2010), 105-126.

(Keith Kearnes) Department of Mathematics, University of Colorado, Boulder, CO 80309-0395, USA

E-mail address: Keith.Kearnes@Colorado.EDU

(Emil W. Kiss) Loránd Eötvös University, Department of Algebra and Number Theory, 1117 Budapest, PÁzmány PÉter sétány 1/C, Hungary

E-mail address: ewkiss@cs.elte.hu

(Ágnes Szendrei) Department of Mathematics, University of Colorado, Boulder, CO 80309-0395, USA

E-mail address: Agnes.Szendrei@Colorado.EDU 